\title{
Rigorous Derivation of the Pauli Equation with Time- dependent Electromagnetic Field
}

\author{
NORBERT J. MAUSER* \\ Univ. Wien, Inst. f. Math., Strudlhofg.4, A-1090 Wien, Austria and Univ. Nice, Lab. J.A. Dieudonné, \\ URM 6621 du CNRS, Parc Valrose, F-06108 Nice, France
}

(Received 13 August 1997; In final form 1 December 1998)

\begin{abstract}
In this work we discuss relativistic corrections for the description of charge carriers in a quantum mechanical framework. The fundamental equation is the Dirac equation which takes into account also the electron's spin. However, this equation intrinsically also incorporates positrons which play no role in applications in solid state physics. We give a rigorous derivation of the Pauli equation describing electrons in a first order approximation of the Dirac equation in the limit of infinite velocity of light. We deal with time-dependent electromagnetic potentials where no rigorous results have been given before. Our approach is based on the use of appropriate projection operators for the electron and the positron component of the spinor which are better suited than the widely used simple splitting into 'upper (large)' and 'lower (small) component'. We also systematically derive corrections at second order in $1 / c$ where we essentially recover the results of the Foldy-Wouthuysen approach. However, due to the non-static problem, differences occur in the term which couples the electric field with the spin.
\end{abstract}

Keywords: Relativistic quantum transport, Dirac equation, Pauli equation, relativistic corrections, nonrelativistic limit, Foldy-Wouthuysen transformation

\section{INTRODUCTION}

Quantum effects play an important role in some technologically important semiconductor devices such as the resonant tunneling diode. The basic description of charge carriers is hence given by Schrödinger equations or their kinetic counterpart, Wigner equations. Under certain conditions also relativistic effects cannot be neglected $[2,4]$ - like e.g., for heavy hydrogenic elements. The fundamental quantum mechanical and relativistic equation is the Dirac-Maxwell system, i.e., the Dirac equation [5] for the electron as a spinor coupled to the Maxwell equations for the electromagnetic field. However, this system is very tedious to deal with both analytically and numerically - for example global existence with arbitrary initial data is still an open problem. In this work we deal with

*e-mail: mauser@cma.univie.ac.at 
the linear case, i.e., the case where the electromagnetic potential is given, e.g., the situation of a single electron in an external electromagnetic field. This is consistent with our assumption of bounded potentials which would not hold for the case of the selfconsistent Coulomb interaction among an ensemble of electrons. However, we are able, for the first time, to deal with the general case of time dependent potentials.

The Pauli equation is an approximation of the Dirac equation in two respects: on the one hand it is basically a first order approximation (in $1 / c$ ), on the other hand it is an equation for the 2 spinor of the electron component of the 4 spinor of the Dirac equation that contains also a positron component which can be neglected in most problems of solid state physics.

The Dirac equation for a relativistic particle with spin in a given electromagnetic field is given by

$$
\begin{aligned}
i \partial_{t} \psi= & -i c \gamma^{0} \gamma^{k} \partial_{k} \psi+\frac{m c^{2}}{\hbar} \gamma^{0} \psi \\
& -\frac{q}{\hbar} A_{k} \gamma^{0} \gamma^{k} \psi-\frac{q}{\hbar} A_{0} \psi
\end{aligned}
$$

The unknown $\psi$ is the 4-vector of the 'Spinorfield' $: \psi(t, x) \in \mathbb{C}, x_{0}=c t \in \mathbb{R}, x=\left(x_{1}, x_{2}, x_{3}\right) \in \mathbb{R}^{3} . \partial_{\mu}$ stands for $\left(\partial / \partial x_{\mu}\right)$, i.e. $\left(\partial_{0}=\partial / \partial x_{0}\right), \partial_{k}=\left(\partial / \partial x_{k}\right)$, where the greek letter $\mu$ stands for $0,1,2,3$ and $k$ denotes the 3 spatial dimension indices 1, 2, 3. The summation convention for indices that appear as 'a pair co- and contravariant indices' is consequently used, e.g., $\gamma^{\mu} A_{\mu}$ stands for $\sum_{\mu=0}^{3} \gamma^{\mu} A_{\mu}$.

The Dirac matrices $\gamma^{\mu}=\mathbb{C}^{4 \times 4}, \mu=0, \ldots, 3$, and the Pauli matrices $\sigma^{k} \in \mathbb{C}^{2 \times 2}, k=1,2,3$ are given by

$$
\begin{gathered}
\gamma^{0}=\left(\begin{array}{cccc}
1 & & & \\
& 1 & & \\
& & -1 & \\
& & -1
\end{array}\right), \quad \gamma^{k}=\left(\begin{array}{cc}
0 & \sigma^{k} \\
-\sigma^{k} & 0
\end{array}\right) \\
\sigma^{1}=\left(\begin{array}{ll}
0 & 1 \\
1 & 0
\end{array}\right), \quad \sigma^{2}=\left(\begin{array}{cc}
0 & -i \\
i & 0
\end{array}\right), \\
\sigma^{3}=\left(\begin{array}{cc}
1 & 0 \\
0 & -1
\end{array}\right)
\end{gathered}
$$

In the sequel also the following related matrices occur frequently

$$
\gamma^{0} \gamma^{k}=\left(\begin{array}{cc}
0 & \sigma^{k} \\
\sigma^{k} & 0
\end{array}\right), S^{m}:=i \gamma^{k} \gamma^{l}=\left(\begin{array}{cc}
\sigma^{m} & 0 \\
0 & \sigma^{m}
\end{array}\right)
$$

where $(k, l, m)$ are cyclic permutations of $(1,2,3)$. Note that the matrices $S^{m}$ represent the 'spinoperator' [11].

$A_{\mu}(t, x) \in \mathbb{R}^{3}, \mu=0, \ldots, 3$, are the components of the time-dependent electromagnetic potential, in particular $A_{0}(t, x)$ is the electric potential and $\vec{A}(t, x)=\left(A_{1}, A_{2}, A_{3}\right)^{\top}$ is the magnetic potential vector. Hence the electric field and the magnetic field are given by

$$
\vec{E}(t, x)=\vec{\nabla} A_{0}-\partial_{t} \vec{A} \quad \vec{B}(t, x)=\operatorname{curl} \vec{A} .
$$

The physical constants are $M=m_{0} c / \hbar, g=e / \hbar$, where $m_{0}$ is the electron's rest mass, $c$ is the velocity of light, $\hbar$ is the Planck constant and $e$ is the unit charge.

There are two physically important limits of the Dirac equation. The 'classical limit' $\hbar \rightarrow 0$ with $c$ fixed, which was rigorously performed e.g., in [8] using Wigner transform techniques. Similar techniques were used in [1] for the classical limit of a Pauli equation. The 'nonrelativistic limit' $c \rightarrow \infty$ is the limit where the velocity of light tends to infinity and 'instaneous interactions' are considered.

The following graph shows the relation between the linear Dirac equation and it's approximations in these limits.

In this work we focus on the limit $c \rightarrow \infty$, in particular on a mathematically rigorous study of the relation between the Dirac and the Pauli equation. First we rescale Eq. (1.1) properly ([3]) and introduce the dimensionless parameter $\varepsilon$ as the inverse of the speed of light $c$ :

$$
\varepsilon \simeq 1 / c
$$

The resulting scaled Dirac equation for $\psi^{\varepsilon}=\psi^{\varepsilon}(t, x)$ reads

$$
\begin{aligned}
i \partial_{t} \psi^{\varepsilon}= & -\frac{i}{\varepsilon} \gamma^{0} \gamma^{k} \partial_{k} \psi^{\varepsilon}+\frac{1}{\varepsilon^{2}} \gamma^{0} \psi^{\varepsilon} \\
& -A_{k} \gamma^{0} \gamma^{k} \psi^{\varepsilon}-A_{0} \psi^{\varepsilon}
\end{aligned}
$$




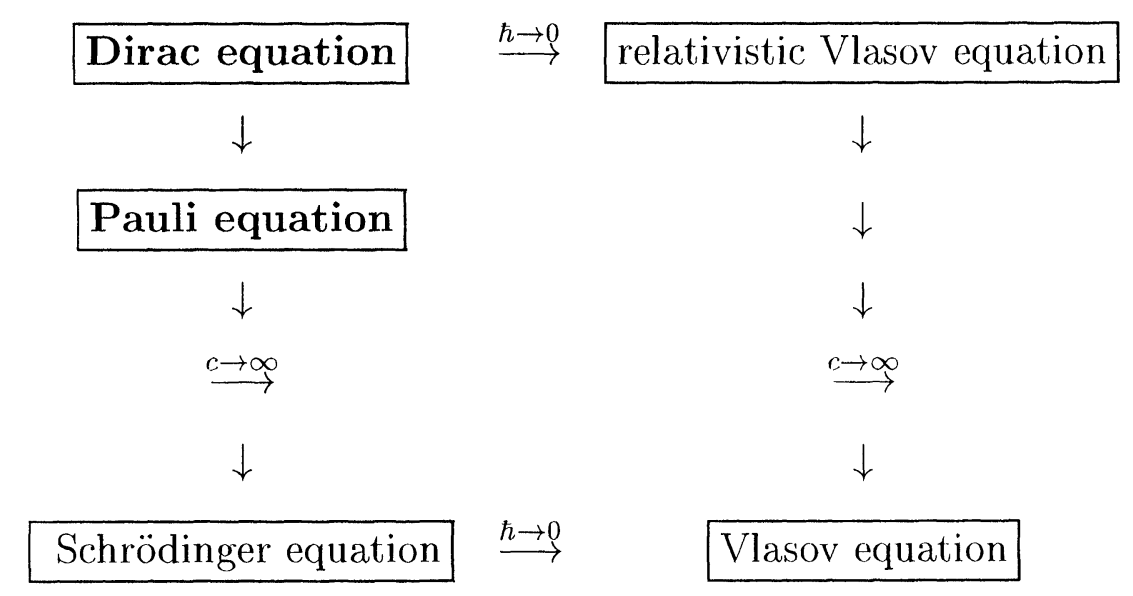

For the nonlinear Dirac-Maxwell system we have the following relations

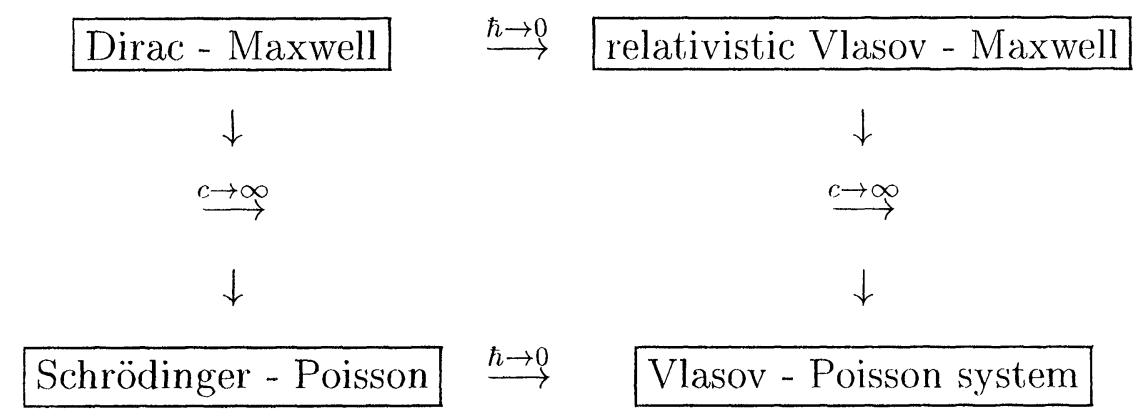

$$
\psi^{\varepsilon}(t=0, x)=\psi_{I}^{\varepsilon}(x)
$$

By 'semi-nonrelativistic approximations' we denote approximations which retain terms at least at $O(\varepsilon)$ and we reserve the name 'Pauli equation' for equations 'at first order in $\varepsilon$ ', containing in any case the spin-magnetic field coupling term with the famous factor $1 / 2$ as in (2.8). The 'total' nonrelativistic limit $\varepsilon \rightarrow 0$ yields 'Schrödinger equations' where the spin only rests as a divergence free term in the current density and, in our scaling, the magnetic field has vanished (in contrast to the scaling e.g., in (the works based on) [9]) where the relativistic dynamics of the electron are somewhat decoupled of the relativistic nature of the magnetic field.

Foldy and Wouthuysen (F-W) [7] have given the first systematic approach to (semi)-nonrelativistic approximations which still rests more or less heuristic. A mathematically rigorous theory of the problem has been developed based on a pseudoresolvent convergence approach using the spectral theorem, e.g. [9, 14]. For a general survey with an exhausting list of references see [13]. All these results, however, treat the mere static case, i.e., time-independent electromagnetic potentials.

In contrast, we use very direct functional analytic methods for rigorously deriving the Pauli equation for time-dependent potentials and studying also the $O\left(\varepsilon^{2}\right)$ approximation. Our rigorous approach is somewhat related to the F-W approach which is essentially the search for a unitary transformation which diagonalizes the Dirac Hamiltonian with respect to the orthogonal decomposition based on $\Pi_{ \pm}^{0}$. The decomposition based on the PDO projectors $\Pi_{ \pm}^{\varepsilon}(D)$ is better 
suited which is reflected e.g., by the fact the resulting 'positron-small component' is $O\left(\varepsilon^{2}\right)$ in contrast to the $O(\varepsilon)$ 'lower-small component' (2.6) obtained via the $\Pi_{ \pm}^{0}$ decomposition.

In our scaling the position density $n^{\varepsilon}(t, x)$ is given by

$$
n^{\varepsilon}(t, x)=J_{0}^{\varepsilon}(t, x)=\psi^{\varepsilon}(t, x) \cdot \overline{\psi^{\varepsilon}}(t, x) .
$$

and the components $J_{k}^{\varepsilon}$ of the 3 -vector $\vec{J}^{\varepsilon}$ of the current density by

$$
J_{k}^{\varepsilon}(t, x)=\frac{1}{\varepsilon} \gamma^{0} \gamma^{k} \psi^{\varepsilon}(t, x) \cdot \overline{\psi^{\varepsilon}}(t, x)
$$

Rigorous results for nonrelativistic approximations of the current density which are seldom studied in the mathematical literature in this field are given in [3].

We denote by $D=\left(D_{1}, D_{2}, D_{3}\right)$ the spatial derivative corresponding to the Fourier multiplier $\xi$ and by $D_{k}=-i \partial_{k}$ the partial derivative associated to $\xi_{k}$.

We define the "free Dirac operator" $Q^{\varepsilon}$ and the "electromagnetic operator" $\mathcal{A} \equiv \mathcal{A}(t, x)$

$$
\begin{array}{r}
Q^{\varepsilon}(D):=\varepsilon \gamma^{0} \gamma^{k} D_{k}+\gamma^{0} I d, \\
\mathcal{A}:=A_{k}(t, x) \gamma^{0} \gamma^{k}+A_{0}(t, x) I d,
\end{array}
$$

and rewrite (1.1) as

$$
i \partial_{t} \psi^{\varepsilon}=\frac{1}{\varepsilon^{2}} Q^{\varepsilon} \Psi^{\varepsilon}+\mathcal{A} \psi^{\varepsilon} .
$$

The spectral problem for the self-adjoint operator $Q^{\varepsilon}$ on Fourier space, i.e., for $Q^{\varepsilon}(\xi)=\varepsilon \gamma^{0} \gamma^{k} \xi_{k}+$ $\gamma^{0} I d$, shows that for each $\xi$ there are 2 eigenvalues $\pm \lambda^{\varepsilon}$ of $Q^{\varepsilon}(\xi)$ with geometric multiplicity 2 given by

$$
\lambda^{\varepsilon}(\xi):=\sqrt{1+\varepsilon^{2}|\xi|^{2}}
$$

The associated projectors $\Pi_{ \pm}^{\varepsilon}(\varepsilon)$ are easily calculated and correspond to the pseudo differential operators $\Pi_{ \pm}^{\varepsilon}(D)$ on $L^{2}\left(\mathbb{R}_{x}^{3}\right)^{4}$

$$
\Pi_{ \pm}^{\varepsilon}(D)=\frac{1}{2}\left(I d \pm \frac{Q^{\varepsilon}(D)}{\lambda^{\varepsilon}(D)}\right)
$$

By means of these projectors we can decompose $L^{2}\left(\mathbb{R}_{x}^{3}\right)^{4}$ in two subspaces corresponding to free electrons and positrons ([5]), since the positive and negative eigenvalues $\pm \lambda^{\varepsilon}$ correspond to positive and negative energies of a free Dirac particle [5].

In the formal limit $\varepsilon \rightarrow 0$ of $\Pi_{ \pm}^{\varepsilon}(D)$ we obtain the operators

$$
\begin{aligned}
& \Pi_{ \pm}^{0}=\frac{1}{2}\left(I \pm \gamma^{0}\right), \\
& \Pi_{+}^{0}=\left(\begin{array}{llll}
1 & & & \\
& 1 & & \\
& & 0 & \\
& & & 0
\end{array}\right), \\
& \Pi_{-}^{0}=\left(\begin{array}{llll}
0 & & & \\
& 0 & & \\
& & 1 & \\
& & & 1
\end{array}\right)
\end{aligned}
$$

The projectors $\Pi_{ \pm}^{\varepsilon}(D)$ are uniformly (in $\varepsilon$ ) bounded operators from $H^{m}\left(\mathbb{R}^{3}\right)^{4} \rightarrow H^{m}\left(\mathbb{R}^{3}\right)^{4}$ and by calculation in Fourier space we verify the following series expansion of $\Pi_{ \pm}^{\varepsilon}(D)$ (w.r.t. $\varepsilon$ ):

$$
\Pi_{ \pm}^{\varepsilon}(D)=\Pi_{ \pm}^{0} \mp i \frac{\varepsilon}{2} \gamma^{0} \gamma^{k} \partial_{k} \pm \frac{\varepsilon^{2}}{4} \gamma^{0} \triangle \pm \varepsilon^{4} R_{4}
$$

where $R_{4}$ stands for a uniformly (in $\varepsilon$ ) bounded operator $H^{m}\left(\mathbb{R}^{3}\right)^{4} \rightarrow H^{m-4}\left(\mathbb{R}^{3}\right)^{4}$.

In this paper we present different methods to derive the Pauli equation (2.8) as well as higher order approximations. We first present a textbook derivation of the Pauli equation for the 2-spinor of the electron component. Then we show how to obtain an implicitly equivalent equation for 4spinors by a simple formal Hilbert expansion in $\varepsilon$ 
based on our special definition of the electron-large and position-small component. Tis development in $\varepsilon$ yields also higher order corrections in a more direct way than the Foldy-Wouthousen approach. After justifying rigorously our derivation of the Pauli equation and connecting it to the usual one we discuss in particular the second order terms more closely.

\section{THE "LARGE VS. SMALL COMPONENT" APPROACH AND FORMAL DERIVATIONS}

The above 'algebraic' projection operators (1.16) are employed for the standard derivation of the Pauli equation via the "large and small component" approach, i.e., the splitting of the "Dirac 4spinor" into two "Pauli 2-spinors" as follows (e.g. $[6,10,11])$ :

Defining the "upper" and "lower component" as

$$
\psi_{l}^{\varepsilon}(t, x):=\Pi_{+}^{0} \psi^{\varepsilon}(t, x), \quad \psi_{s}^{\varepsilon}(t, x):=\Pi_{-}^{0} \psi^{\varepsilon}(t, x)
$$

we have, of course,

$$
\psi^{\varepsilon}=\left(\begin{array}{c}
\psi_{1}^{\varepsilon} \\
\psi_{2}^{\varepsilon} \\
\psi_{3}^{\varepsilon} \\
\psi_{4}^{\varepsilon}
\end{array}\right)=\left(\begin{array}{c}
\psi_{1}^{\varepsilon} \\
\psi_{2}^{\varepsilon} \\
0 \\
0
\end{array}\right)+\left(\begin{array}{c}
0 \\
0 \\
\psi_{3}^{\varepsilon} \\
\psi_{4}^{\varepsilon}
\end{array}\right)=\psi_{l}^{\varepsilon}+\psi_{s}^{\varepsilon}
$$

Introducing $\widetilde{\psi_{l}^{\varepsilon}}$ and $\widetilde{\psi_{s}^{\varepsilon}}$ as the vectors with two components skipping the zeros in $\psi_{l}^{\varepsilon}, \psi_{s}^{\varepsilon}$ the Dirac equation can be split two equations for 2-vectors involving the Pauli matrices. Defining the "upperlarge" and the "lower-small component" as the 2vectors

$$
\begin{aligned}
& \varphi_{l}^{\varepsilon}(t, x):=\mathrm{e}^{i t / \varepsilon^{2}} \widetilde{\psi_{l}^{\varepsilon}}(t, x), \\
& \varphi_{s}^{\varepsilon}(t, x):=\mathrm{e}^{i t / \varepsilon^{2}} \widetilde{\psi_{s}^{\varepsilon}}(t, x)
\end{aligned}
$$

we immediately obtain the equations

$$
\begin{aligned}
i \partial_{t} \varphi_{l}^{\varepsilon} & =-i \frac{1}{\varepsilon} \sigma^{k} \partial_{k} \varphi_{s}^{\varepsilon}-A_{k} \sigma^{k} \varphi_{s}^{\varepsilon}-A_{0} \varphi_{l}^{\varepsilon} \\
i \partial_{t} \varphi_{s}^{\varepsilon} & =-i \frac{1}{\varepsilon} \sigma^{k} \partial_{k} \varphi_{l}^{\varepsilon}-A_{k} \sigma^{k} \varphi_{l}^{\varepsilon}-A_{0} \varphi_{s}^{\varepsilon}-\frac{2}{\varepsilon^{2}} \varphi_{s}^{\varepsilon}
\end{aligned}
$$

By formal consideration of orders of magnitude, in particular assuming that $\partial_{t} \varphi_{s}^{\varepsilon}$ is of $O(1)$, we obtain from (2.5) that the lower-small component is $O(\varepsilon)$

$$
\varphi_{s}^{\varepsilon}=-\frac{i}{2} \varepsilon \sigma^{k} \partial_{k} \varphi_{l}^{\varepsilon}+O\left(\varepsilon^{2}\right)
$$

and for the upper-large component we obtain by using (2.6) in (2.4)

$$
\begin{aligned}
i \partial_{t} \varphi_{l}^{\varepsilon}= & -\frac{1}{2} \sigma^{k} \sigma^{l} \partial_{k} \partial_{l} \varphi_{l}^{\varepsilon}-A_{0} \varphi_{l}^{\varepsilon} \\
& +\frac{i}{2} \sigma^{k} \sigma^{l} \partial_{k} A_{l} \varphi_{l}^{\varepsilon}+i A_{k} \partial_{k} \varphi_{l}^{\varepsilon}+O\left(\varepsilon^{2}\right)
\end{aligned}
$$

Using the properties of the Pauli matrices $\sigma^{j}$ and adding the $O\left(\varepsilon^{2}\right)$ term $\varepsilon^{2}|\vec{A}|^{2}$, where $\vec{A}:=\left(A_{1}\right.$, $\left.A_{2}, A_{3}\right)$ is the magnetic potential, this formal procedure finally yields the "Pauli equation" $[10,11]$ for $\tilde{\varphi}_{l}^{\varepsilon}$ as the " $O(\varepsilon)$ approximation" of the upper-large component $\varphi_{l}^{\varepsilon}$.

$$
i \partial_{t} \tilde{\varphi}_{l}^{\varepsilon}=(i \vec{\nabla}+\varepsilon \vec{A})^{2} \tilde{\varphi}_{l}^{\varepsilon}-A_{0} \tilde{\varphi}_{l}^{\varepsilon}-\varepsilon \frac{1}{2} \sigma^{k} B_{k} \tilde{\varphi}_{l}^{\varepsilon}
$$

Here and in the sequel the tilde $\sim$ denotes the solution of an approximative equation i.e., $\tilde{\varphi}_{l}^{\varepsilon}$ is the solution of the approximation (2.8) obtained by neglecting $O\left(\varepsilon^{2}\right)$ terms in the exact Eq. (2.7).

In contrast to the above approach of "upperlarge and lower-small component" by applying the "algebraic projects" $\Pi_{ \pm}^{0}$ to (1.7), our mathematically rigorous theory is based on the use of the "pseudo differential operator projectors" $\Pi_{ \pm}^{\varepsilon}(D)$. This implies that the "small component" $\Pi_{-}^{\varepsilon} \phi$ is of $O\left(\varepsilon^{2}\right)$ in $L^{\infty}\left((0, T) ; H^{m}\left(\mathbb{R}^{3}\right)^{4}\right)$ and not of $O(\varepsilon)$ as in (2.6) and the magnetic field appears in the small component at leading order. In addition, the "Pauli equation" becomes an equation for a 4vector with an additional term at $O(\varepsilon)$. However, 
we can reformulate this Pauli equation by an equation for a 2-vector in the usual form (2.8).

We define the "electron-large component" $\phi_{+}^{\varepsilon}$ and the "positron-small component" $\phi_{-}^{\varepsilon}$ as follows: The "electron" $\psi_{+}^{\varepsilon}$ and "positron component" $\psi_{-}^{\varepsilon}$ are introduced via the PDO-projectors $\Pi_{ \pm}^{\varepsilon}(D)(1.15)$ and like in (2.3) we "factor out" the rest energy $m_{0} c^{2}$ i.e., $1 / \varepsilon^{2}$ in our scaling:

$$
\begin{aligned}
\phi^{\varepsilon}(t, x) & :=\mathrm{e}^{i t / \varepsilon^{2}} \psi^{\varepsilon}(t, x) \\
& =\phi_{+}^{\varepsilon}(t, x)+\phi_{-}^{\varepsilon}(t, x) \quad \text { with } \\
\phi_{+}^{\varepsilon}(t, x): & =\Pi_{+}^{\varepsilon} \phi^{\varepsilon}(t, x)=\mathrm{e}^{i t / \varepsilon^{2}} \psi_{+}^{\varepsilon}(t, x), \\
\phi_{-}^{\varepsilon}(t, x): & =\Pi_{-}^{\varepsilon} \phi^{\varepsilon}(t, x)=\mathrm{e}^{i t / \varepsilon^{2}} \psi_{-}^{\varepsilon}(t, x)
\end{aligned}
$$

Note that the use of the same sign in the exponential means that we subtract the positive rest energy of the electron component and add the negative rest energy of the positron component (as apparent e.g., in (2.12)). This is the basis of any "large" vs. "small" component approach.

Applying the projectors $\Pi_{ \pm}^{\varepsilon}$ to (1.13) and using (2.10) yields a splitting of the Dirac equation

$$
\begin{aligned}
& i \partial_{t} \phi_{+}^{\varepsilon}-\frac{\lambda^{\varepsilon}-1}{\varepsilon^{2}} \phi_{+}^{\varepsilon}+\Pi_{+}^{\varepsilon}\left(\mathcal{A} \phi^{\varepsilon}\right)=0 \quad \text { and } \\
& i \partial_{t} \phi_{-}^{\varepsilon}+\frac{\lambda^{\varepsilon}+1}{\varepsilon^{2}} \phi_{-}^{\varepsilon}+\Pi_{-}^{\varepsilon}\left(\mathcal{A} \phi^{\varepsilon}\right)=0
\end{aligned}
$$

Since we have used the same sign for the phase factors in the transformation (2.10) we have broken the symmetry between electrons and positrons. This asymmetric becomes transparent in a development of the "kinetic energy PDO" in (2.11)

$$
\begin{aligned}
& \frac{\lambda^{\varepsilon}(D)-1}{\varepsilon^{2}}=\frac{1}{2} \Delta+\varepsilon^{2} R \text { and } \\
& \frac{\lambda^{\varepsilon}(D)+1}{\varepsilon^{2}}=\frac{2}{\varepsilon^{2}} I d+\frac{1}{2} \Delta+\varepsilon^{2} R
\end{aligned}
$$

where $R$ stands for an unspecified uniformly (w.r.t. $\varepsilon$ ) bounded operator $H^{m}\left(\mathbb{R}^{3}\right)^{4} \rightarrow H^{m-4}\left(\mathbb{R}^{3}\right)^{4}$. The difference between the two operators is precisely twice the positron's rest energy.

Of course, in the expansion of PDOs like in (2.12) we "lose regularity for every power of $\varepsilon$ " which makes the reminder terms more and more singular. However, assuming sufficient regularity of the initial data and the electromagnetic potential we can always counterbalance in order to avoid distributional spaces. This is based on the following "conservation of regularity" of the Dirac equation with time-dependent electromagnetic potential $A$ which holds also for $\phi^{\varepsilon}$ since it differs from $\psi^{\varepsilon}$ be a mere phase factor in time. It is proved by straightforward multiplication and integration of (1.7).

\section{Lemma 2.1 Conservation of Regularity}

(i) Let $A=\left(A_{0}, A_{1}, A_{2}, A_{3}\right) \in L^{\infty}\left((0, T) ; W^{m, \infty}\right.$ $\left.\left(\mathbb{R}^{3}\right)^{4}\right)$ and $\psi_{I}^{\varepsilon}=O(1) \in H^{m}\left(\mathbb{R}^{3}\right)^{4}$.

Then the solution $\psi^{\varepsilon}(t, x)$ of the Dirac equation (1.7) and its transformed $\phi^{\varepsilon}(t, x)$ as defined in (2.9) satisfy

$$
\begin{aligned}
& \left\|\phi^{\varepsilon}\right\|_{L^{\infty}\left((0, T) ; H^{m}\left(\mathbb{R}^{3}\right)^{4}\right)}= \\
& \left\|\psi^{\varepsilon}\right\|_{L^{\infty}\left((0, T) ; H^{m}\left(\mathbb{R}^{3}\right)^{4}\right)} \leq C
\end{aligned}
$$

where $c$ is independent of $\varepsilon$.

(ii) Let $A=\left(A_{0}, A_{1}, A_{2}, A_{3}\right) \in W^{1, \infty}\left((0, T) ; W^{m, \infty}\right.$ $\left(\mathbb{R}^{3}\right)^{4}, \psi_{I}^{\varepsilon}=\phi^{\varepsilon}(t=0)=O(1) \in H^{m+1}\left(\mathbb{R}^{3}\right)^{4}$.

Then the solution $\phi^{\varepsilon}(t, x)=\phi_{+}^{\varepsilon}(t, x)+$ $\phi_{-}^{\varepsilon}(t, x)(c p .(2.9))$ of the "split Dirac equation" (2.11) satisfies

$$
\partial_{t} \phi^{\varepsilon} \in L^{\infty}\left((0, T) ; H^{m}\left(\mathbb{R}^{3}\right)^{4}\right)
$$

where $C_{T}$ is an $\varepsilon$-independent constant depending on the time intervall $(0, T)$.

The "matrix PDO projects" $\Pi_{ \pm}^{\varepsilon}(D)$ do not commute with the ( $x$-dependent) "matrix electromagnetic operator" $\mathcal{A}(t, x)$ occurring in (2.11). A development of the commutators up to second order in $\varepsilon$ is obtained by direct calculation:

$$
\begin{aligned}
\Pi_{ \pm}^{\varepsilon}\left(A_{k} \gamma^{0} \gamma^{k} \phi^{\varepsilon}\right)= & A_{k} \gamma^{0} \gamma^{k} \Pi_{\mp}^{\varepsilon} \phi^{\varepsilon} \\
& \mp i \varepsilon\left(A_{k} \partial_{k} \phi^{\varepsilon}+\frac{1}{2} \gamma^{l} \gamma^{k}\left(\partial_{l} A_{k}\right) \phi^{\varepsilon}\right) \\
& \pm \frac{\varepsilon^{2}}{4} \gamma^{k}\left\{\Delta\left(A_{k} \phi^{\varepsilon}\right)+A_{k} \Delta \phi^{\varepsilon}\right\} \\
& \pm \varepsilon^{3} R_{3}\left(A_{k} \phi^{\varepsilon}\right)
\end{aligned}
$$




$$
\begin{aligned}
\Pi_{ \pm}^{\varepsilon}\left(A_{0} \phi^{\varepsilon}\right)= & A_{0} \Pi_{ \pm}^{\varepsilon} \phi^{\varepsilon} \mp \frac{i}{2} \varepsilon \gamma^{0} \gamma^{k}\left(\partial_{k} A_{0}\right) \phi^{\varepsilon} \\
& \pm \frac{\varepsilon^{2}}{4} \gamma^{0}\left\{\Delta\left(A_{0} \phi^{\varepsilon}\right)-A_{0} \Delta \phi^{\varepsilon}\right\} \\
& \pm \varepsilon^{3} R_{3}\left(A_{0} \phi^{\varepsilon}\right)
\end{aligned}
$$

where $R_{3}$ stands for an unspecified, uniformly (in $\varepsilon$ ) bounded operator $H^{m}\left(\mathbb{R}^{3}\right)^{4} \rightarrow H^{m-4}\left(\mathbb{R}^{3}\right)^{4}$.

We can obtain approximation of the split Dirac equation (2.11) at any order in $\varepsilon$ a formal way by defining the Hilbert expansions for $\phi_{+}^{\varepsilon}, \phi_{-}^{\varepsilon}$ :

$$
\phi_{+}^{\varepsilon}=\sum_{k=0}^{\infty} \varepsilon^{k} \phi_{+}^{k}, \quad \phi_{-}^{\varepsilon}=\sum_{k=0}^{\infty} \varepsilon^{k} \phi_{-}^{k}
$$

Note that $\phi_{+}^{k}, \phi_{-}^{k}$ are 4 -spinors for all $k$. By plugging (2.17) into (2.11) combined with expansions like (2.15), (2.16), (2.12) we can proceed by formally comparing orders of $\varepsilon$.

We immediately see that the positron-small component starts at $O\left(\varepsilon^{2}\right)$, i.e., $\phi_{-}^{0}=0, \phi_{-}^{1}=0$ and we can easily calculate the terms of each order in $\varepsilon$

$O(1)$ :

$$
i \partial_{t} \phi_{+}^{0}=-\frac{\Delta}{2} \phi_{+}^{0}-A_{0} \phi_{+}^{0}
$$

$O(\varepsilon)$

$$
\begin{aligned}
i \partial_{t} \phi_{+}^{1}= & -\frac{\Delta}{2} \phi_{+}^{1}-A_{0} \phi_{+}^{1}+\frac{i}{2} \partial_{k} A_{l} \gamma^{k} \gamma^{l} \phi_{+}^{0} \\
& -i A_{k} \partial_{k} \phi_{+}^{0}-\frac{i}{2} \gamma^{0} \gamma^{k} \partial_{k} A_{0} \phi_{+}^{0}
\end{aligned}
$$

Defining $\phi_{P}^{\varepsilon}:=\phi_{+}^{0}+\varepsilon \phi_{+}^{1}$ we immediately see that $\phi_{P}^{\varepsilon}$ fulfills Eq. (2.19) by adding up the Eq. (2.18) and (2.19) and using $\phi_{+}^{0}=\phi_{P}^{\varepsilon}+O(\varepsilon)$ :

$$
\begin{aligned}
i \partial_{t} \phi_{P}^{\varepsilon}= & -\frac{\Delta}{2} \phi_{P}^{\varepsilon}-A_{0} \phi_{P}^{\varepsilon}+\varepsilon \frac{i}{2} \partial_{k} A_{l} \gamma^{k} \gamma^{l} \phi_{P}^{\varepsilon} \\
& -\varepsilon i A_{k} \partial_{k} \phi_{P}^{\varepsilon}-\varepsilon \frac{i}{2} \gamma^{0} \gamma^{k} \partial_{k} A_{0} \phi_{P}^{\varepsilon}+O\left(\varepsilon^{2}\right)
\end{aligned}
$$

We can rewrite (2.20) using the algebra of the Dirac matrices $\gamma^{j}$ : the diagonal in the double sum of the third term on the r.h.s. of (2.20) gives $-\frac{i}{2}\left(\partial_{k} A_{k}\right) \phi_{P}^{\varepsilon}$ and the off diagonal in this term yields $1 / 2 S^{m} \operatorname{curl}_{m} \vec{A} \phi_{P}^{\varepsilon}=1 / 2 S^{m} B_{m} \phi_{P}^{\varepsilon}$, where the "block-diagonal" matrix $S^{m}$ is given by (1.4).

Adding the $O(\varepsilon)^{2}$ term $\varepsilon^{2}|\vec{A}|^{2}$, where $\vec{A}:=\left(A_{1}\right.$, $\left.A_{2}, A_{3}\right)$ is the magnetic potential, we see that the following equation holds for $\phi^{\varepsilon}=\phi_{P}^{\varepsilon}$ :

Definition 2.1 The "Pauli equation for 4-spinors" is given by

$$
\begin{aligned}
i \partial_{t} \phi^{\varepsilon}= & (i \nabla+\varepsilon \vec{A})^{2} \phi^{\varepsilon}+A_{0} \phi^{\varepsilon}-\varepsilon \frac{1}{2} S^{k} B_{k} \phi^{\varepsilon} \\
& +\varepsilon \frac{i}{2} \gamma^{0} \gamma^{k}\left(\partial_{k} A_{0}\right) \phi^{\varepsilon}
\end{aligned}
$$

The third term in (2.21) contains the "block-antidiagonal" matrices $\gamma^{0} \gamma^{k}(c f$. (1.4)). This term plays a special role as we shall see in the follow. Note that $\partial_{k} A_{0}=E_{k}-\partial_{t} A_{k}$ and only in the case of a static magnetic field this term involves the mere electric field. This is a difference to the analogous term found in $[5,11,12]$. We note that $i \gamma^{0} \gamma^{k}\left(\partial_{k} A_{0}\right)$ is anti-selfadjoint since $\gamma^{0} \gamma^{k}$ are self-adjoint and $\partial_{k} A_{0}$ is real.

This way we find the " $O(\varepsilon)$ approximation" of the electron-large component $\phi_{+}^{\varepsilon}$ by the 4-spinor $\phi_{P}^{\varepsilon}$ as the solution of the Pauli equation (2.21) with proper initial data $\phi_{P}^{\varepsilon}(t=0, x)=\phi_{P I}^{\varepsilon}(x)$.

\section{THE PAULI EQUATION AS THE "SEMI-NONRELATIVISTIC LIMIT"}

The formal Hilbert expansion that led to Eq. (2.21) can be justified rigorously. The approximation keeping terms at first order, i.e., at $O(\varepsilon) \sim O(1 / c)$, yields the Pauli equation as the "semi-nonrelativistic limit" of the Dirac equation (1.7).

The equations for the electon-large and positronsmall component $\phi_{+}^{\varepsilon}$ and $\phi_{-}^{\varepsilon}$ as defined in (2.10) are readily obtained from (2.11) using (2.12) and (2.15), (2.16):

$$
\begin{aligned}
i \partial_{t} \phi_{+}^{\varepsilon}+ & \frac{\Delta}{2} \phi_{+}^{\varepsilon}+A_{0} \phi_{+}^{\varepsilon}+A_{k} \gamma^{0} \gamma^{k} \phi_{-}^{\varepsilon} \\
& +\varepsilon G \phi^{\varepsilon}=\varepsilon^{2} R \phi^{\varepsilon}
\end{aligned}
$$




$$
\begin{aligned}
i \partial_{t} \phi_{-}^{\varepsilon} & -\frac{\Delta}{2} \phi_{-}^{\varepsilon}+\frac{2}{\varepsilon^{2}} \phi_{-}^{\varepsilon}+A_{0} \phi_{-}^{\varepsilon}+A_{k} \gamma^{0} \gamma^{k} \phi_{+}^{\varepsilon} \\
-\varepsilon G \phi^{\varepsilon} & =-\varepsilon^{2} R \phi^{\varepsilon},
\end{aligned}
$$

with $\phi^{\varepsilon}=\phi_{+}^{\varepsilon}+\phi_{-}^{\varepsilon}$. Here $G \phi^{\varepsilon}$ follows from (2.15), (2.16)

$$
\begin{aligned}
G \phi^{\varepsilon}= & -i A_{k} \delta_{k} \phi^{\varepsilon}+\frac{i}{2} \gamma^{l} \gamma^{k}\left(\partial_{l} A_{k}\right) \phi^{\varepsilon} \\
& -\frac{i}{2} \gamma^{0} \gamma^{k}\left(\partial_{k} A_{0}\right) \phi^{\varepsilon}
\end{aligned}
$$

and is a uniformly bounded operator $H^{m}\left(\mathbb{R}^{3}\right)^{4} \rightarrow$ $H^{m-1}\left(\mathbb{R}^{3}\right)^{4}$ for $A \in L^{\infty}\left((0, T) ; W^{m, \infty}\left(\mathbb{R}^{3}\right)^{4} . R\right.$ is the sum of the remainder terms due to (2.12) and (2.15), (2.16), hence it is a uniformly (w.r.t. $\varepsilon$ ) bounded operator $H^{m}\left(\mathbb{R}^{3}\right)^{4} \rightarrow H^{m-4}\left(\mathbb{R}^{3}\right)^{4}$ for $A \in$ $L^{\infty}\left((0, T) ; W^{m-2, \infty}\left(\mathbb{R}^{3}\right)^{4}\right.$.

The use of the "Pauli equation for 4-spinors" (2.21) is made rigorous in

Theorem 3.1 Let $A=\left(A_{0}, A_{1}, A_{2}, A_{3}\right) \in W^{1, \infty}$ $\left((0, T), W^{2, \infty}\left(\mathbb{R}^{3}\right)^{4}\right)$ and take $\psi_{I}^{\varepsilon}=O(1) \in H^{4}\left(\mathbb{R}^{3}\right)^{4}$ and $\Pi_{-}^{\varepsilon} \psi_{I}^{\varepsilon}=O\left(\varepsilon^{2}\right) \in H^{2}\left(\mathbb{R}^{3}\right)^{4}$.

Then the semi-nonrelativistic approximation (i.e., up to terms of $O\left(\varepsilon^{2}\right)$ ) of the Dirac equation is given by the above Pauli equation (2.21) for the 4-vector $\widetilde{\phi_{+}^{\varepsilon}}$ in the following way:

Let $\psi^{\varepsilon}(t, x)$ be the solution of the Dirac equation (1.7) with initial datum $\psi_{I}^{\varepsilon}(x)$ and let $\phi^{\varepsilon}(t, x)=e^{i t / \varepsilon^{2}}$ $\psi^{\varepsilon}(t, x)$ and $\phi_{ \pm}^{\varepsilon}(t, x)=\Pi_{ \pm}^{\varepsilon}(t, x)$. Let $\widetilde{\phi_{+}^{\varepsilon}}$ be the solution of the Pauli equation (2.21) with initial datum $\widetilde{\phi}_{+I}^{\varepsilon}$ such that $\left\|\psi_{I}^{\varepsilon}-\phi_{+I}^{\varepsilon}\right\|_{L^{2}\left(\mathbb{R}^{3}\right)^{4}}=O\left(\varepsilon^{2}\right)$.

Then

(i)

$$
\left\|\phi_{-}^{\varepsilon}(t)\right\|_{L^{2}\left(\mathbb{R}^{3}\right)^{4}}+\left\|\left(\phi^{\varepsilon}-\widetilde{\phi_{+}^{\varepsilon}}\right)(t)\right\|_{L^{2}\left(\mathbb{R}^{3}\right)^{4}} \leq C_{T} \varepsilon^{2}
$$

where $C_{T}$ is an $\varepsilon$-independent constant depending on the time interval $(0, T)$.

(ii) For the positron-small component $\phi_{-}^{\varepsilon}$ with initial datum $\phi_{-I}^{\varepsilon}(x)=\Pi_{-}^{\varepsilon} \psi_{I}^{\varepsilon}(x)$ we have

$$
\phi_{-}^{\varepsilon}=-\varepsilon^{2} \frac{1}{2} A_{k} \gamma^{0} \gamma^{k} \widetilde{\phi_{+}^{\varepsilon}}-i \varepsilon^{2} \partial_{t} \phi_{-}^{\varepsilon}+\varepsilon^{3} r
$$

where $\widetilde{\phi_{+}^{\varepsilon}}$ is the solution of the Pauli equation (2.21) and, $r, 1 / 2 A_{k} \gamma^{0} \gamma^{k} \phi_{+}^{\varepsilon}$ and $\partial_{t} \phi_{-}^{\varepsilon}$ are uniformly (w.r.t. $\varepsilon)$ bounded functions in $L^{\infty}((0, T)$; $\left.L^{2}\left(\mathbb{R}^{3}\right)^{4}\right)$.

Proof Several estimates, in particular on the small component, are fundamental:

For the time derivative first note that $\partial_{t} \phi_{-}^{\varepsilon}$ $(t=0) \in L^{2}\left(\mathbb{R}^{3}\right)^{4}$ which follows immediately from the assumptions on $\psi_{I}^{\varepsilon}$ and $A$ via Eq. (2.11). We then have $\partial_{t} \phi_{-}^{\varepsilon}(t, x) \in L^{\infty}\left((0, T) ; L^{2}\left(\mathbb{R}^{3}\right)^{4}\right.$ with the bound

$$
\begin{gathered}
\left\|\partial_{t} \phi_{-}^{\varepsilon}(t)\right\|_{L^{2}\left(\mathbb{R}^{3}\right)^{4}} \leq C_{T}\|A\|_{W^{1, \infty}\left((0, T) ; L^{\infty}\left(\mathbb{R}^{3}\right)^{4}\right)}\left\|\psi_{I}^{\varepsilon}\right\|_{H^{2}\left(\mathbb{R}^{3}\right)^{4}} \\
+\left\|\partial_{t} \phi_{-}^{\varepsilon}(t=0)\right\|_{L^{2}\left(\mathbb{R}^{3}\right)^{4}}
\end{gathered}
$$

where $C_{T}$ is an $\varepsilon$-independent constant depending on the time interval $(0, T)$.

In order to proof (3.6) we derive (2.11) with respect to $t$, multiply by $\overline{\partial_{t} \phi_{-}^{\varepsilon}}$, take imaginary parts and integrate with respect to $x$. Straightforward calculations based on the selfadjointness and indempotency of $\Pi_{-}^{\varepsilon}$ allow for estimating the resulting equation term by term. Finally integration over time and Lemma 2.1 allow to conclude (3.6).

The next important estimate is

$$
\begin{aligned}
& \left\|\phi^{\varepsilon}\right\|_{W^{1, \infty}\left((0, T) ; H^{2}\left(\mathbb{R}^{3}\right)^{4}\right)} \\
& \leq C_{T}\|A\|_{W^{1, \infty}\left((0, T) ; W^{2, \infty}\left(\mathbb{R}^{3}\right)^{4}\right)}\left\|\psi_{I}^{\varepsilon}\right\|_{H^{2}\left(\mathbb{R}^{3}\right)^{4}} \\
& \quad+\left\|\partial_{t} \phi^{\varepsilon}(t=0)\right\|_{H^{2}\left(\mathbb{R}^{3}\right)^{4}}
\end{aligned}
$$

where $C_{T}$ is an $\varepsilon$-independent constant depending on the time interval $(0, T)$.

The same estimate holds for the components $\phi_{+}^{\varepsilon}, \phi_{-}^{\varepsilon}$, of course. We first note that the analogon of (3.7) holds as an estimate in $L^{2}\left(\mathbb{R}^{3}\right)^{4}$ since we have (3.6) and the analogous estimate for $\partial_{t} \phi_{+}^{\varepsilon}$ follows by exactly the same proof using the selfadjointness of $1-\lambda^{\varepsilon}(D) / \varepsilon^{2}$.

We take (2.11), derive with respect to $t$ and apply $D^{2}$, multiply by $\overline{\partial_{t} D^{2} \phi_{ \pm}^{\varepsilon}}$ take imaginary parts and integrate with respect to $x$. In the resulting equation we carefully estimate all terms, using again that $\lambda^{\varepsilon}(D) \pm 1 / \varepsilon^{2}$ is self-adjoint and the 
idempotency of $\Pi_{ \pm}^{\varepsilon}$. Straightforward calculations and an intergration in time yield

$$
\begin{aligned}
& \left\|\partial_{t} D^{2} \phi_{ \pm}^{\varepsilon}(t)\right\|_{L^{2}\left(\mathbb{R}^{3}\right)^{4}} \\
& \quad \leq C \cdot t\|A\|_{W^{1, \infty}\left((0, T) ; W^{2, \infty}\left(\mathbb{R}^{3}\right)^{4}\right)} \\
& \quad\left(\left\|\psi_{I}^{\varepsilon}\right\|_{H^{2}\left(\mathbb{R}^{3}\right)^{4}}+\left\|\partial_{t} \phi^{\varepsilon}(t=0)\right\|_{H^{1}\left(\mathbb{R}^{3}\right)^{4}}\right) \\
& \quad+\left\|\partial_{t} D^{2} \phi^{\varepsilon}(t=0)\right\|_{L^{2}\left(\mathbb{R}^{3}\right)^{4}}
\end{aligned}
$$

Again we can conclude $\partial_{t} \phi_{-}^{\varepsilon}(t=0) \in H^{2}\left(\mathbb{R}^{3}\right)^{4}$ from the assumption on $\psi_{I}^{\varepsilon}$ and $A$ directly from Eq. (2.11). Since $\left\|\phi^{\varepsilon}(t)\right\|_{H^{2}\left(\mathbb{R}^{3}\right)^{4}}^{2}=\left\|\phi_{+}^{\varepsilon}(t)\right\|_{H^{2}\left(\mathbb{R}^{3}\right)^{4}}^{2}+$ $\left\|\phi_{-}^{\varepsilon}(t)\right\|_{H^{2}\left(\mathbb{R}^{3}\right)^{4}}^{2}$ we can conclude (3.7).

The third estimate is the rigorous justifications of the name "small" component for $\phi_{-}^{\varepsilon}$ and can be interpreted that "very few" positrons stay "very few" in the finite time evolution governed by the Dirac equation (1.1). Note that the our definition (2.10) of the "small" component gives $O\left(\varepsilon^{2}\right)$ whereas the usual definition (2.3) gives only $O(\varepsilon)$.

$$
\left\|\phi_{-}^{\varepsilon}(t)\right\|_{H^{2}\left(\mathbb{R}^{3}\right) 4}=O(\varepsilon)^{2}, \quad \forall t \in(0, T)
$$

We obtain (3.8) by starting with (2.11)

$$
\left(\lambda^{\varepsilon}+1\right) \phi_{-}^{\varepsilon}=-\varepsilon^{2}\left(i \partial_{t} \phi_{-}^{\varepsilon}+\Pi_{-}^{\varepsilon}\left(\mathcal{A} \phi^{\varepsilon}\right)\right) .
$$

We use (2.11) also for $\Delta \phi_{-}^{\varepsilon}$ use $\lambda^{\varepsilon}(D)+1 \geq 2 I d$.

This finally yields an estimation both for $\left\|\phi_{-}^{\varepsilon}(t)\right\|_{L^{2}\left(\mathbb{R}^{3}\right)^{4}}$ and for $\left\|\Delta \phi_{-}^{\varepsilon}(t)\right\|_{L^{2}\left(\mathbb{R}^{3}\right)^{4}}$ which allows to conclude (3.8).

The Pauli equation for the electron-large component and assertion (ii) of Theorem 3.1 are now immediately obtained by using the estimates (3.6), (3.8) in order to estimate the terms in (3.1), (3.2). With our assumptions the functions $G \phi^{\varepsilon}$ and $R \phi^{\varepsilon}$ are uniformly bounded in $\left.L^{\infty}\left((0, T) ; L^{2}(\mathbb{R})^{3}\right)^{4}\right)$ and we can conclude by keeping the leading order terms and adding the $O\left(\varepsilon^{2}\right)$ term $\varepsilon^{2} \vec{A}^{2}$.

In order to prove (ii) we regard the equation for $\delta_{+}$defined as the difference of $\phi_{+}^{\varepsilon}$ and $\widetilde{\phi}_{+}^{\varepsilon}$. We multiply this equation by $\overline{\delta_{+}}$integrate, take imaginary parts and obtain

$$
\frac{d}{d t}\left\|\delta_{+}\right\|_{L^{2}\left(\mathbb{R}^{3}\right)^{4}} \leq C\left(\varepsilon\left\|\delta_{+}\right\|_{L^{2}\left(\mathbb{R}^{3}\right)^{4}}+\varepsilon^{2}\right)
$$

where $C$ is a constant. Applying a Gronwall Lemma to this inequality gives

$$
\left\|\delta_{+}\right\|_{L^{2}\left(\mathbb{R}^{3}\right)^{4}} \leq\left\|\delta_{+I}\right\|_{L^{2}\left(\mathbb{R}^{3}\right)^{4}} \mathrm{e}^{\varepsilon C t}+\varepsilon\left(\mathrm{e}^{\varepsilon C t}-1\right)
$$

which proves (ii).

It rests to connect (2.21) with the usual Pauli equation (2.8), in particular to clarify the relation between the 4-vector $\widetilde{\phi_{+}^{\varepsilon}}$ and the 2-vector $\tilde{\varphi}_{l}^{\varepsilon}$ as in (2.8) and the nature of the last term in (2.21) which looks like a coupling of spin and electric field at $O(\varepsilon)$.

Defining the "upper electron-large" and the "lower electron-large" component as

$$
\begin{aligned}
& \phi_{+l}^{\varepsilon}:=\Pi_{+}^{0} \phi_{+}^{\varepsilon}=\Pi_{+}^{0} \Pi_{+}^{\varepsilon}(D) \phi^{\varepsilon}, \\
& \phi_{+s}^{\varepsilon}:=\Pi_{-}^{0} \phi_{+}^{\varepsilon}=\Pi_{-}^{0} \Pi_{+}^{\varepsilon}(D) \phi^{\varepsilon}
\end{aligned}
$$

(cp. (2.1)) and analogously for the approximations $\widetilde{\phi_{+l}^{\varepsilon}}$ and $\widehat{\phi_{+s}^{\varepsilon}}$ we can split the Pauli equation (2.21). From Theorem 3.1, (ii) we have $\widetilde{\phi_{+l}^{\varepsilon}}=\widetilde{\phi_{+l}^{\varepsilon}}+O\left(\varepsilon^{2}\right)$ in $L^{\infty}\left((0, T) ; L^{2}(\mathbb{R})^{3}\right)^{4}$ and $(2.21)$ becomes

$$
\begin{aligned}
i \partial_{t} \widetilde{\phi_{+l}^{\varepsilon}}= & (i \nabla+\varepsilon \vec{A})^{2} \widetilde{\phi_{+l}^{\varepsilon}}+A_{0} \widetilde{\phi_{+l}^{\varepsilon}} \\
& -\varepsilon \frac{1}{2} S^{k} B_{k} \widetilde{\phi_{+l}^{\varepsilon}}-i \frac{\varepsilon}{2} S^{k}\left(\partial_{k} A_{0}\right) \widetilde{\phi_{+s}^{\varepsilon}} \\
i \partial_{t} \widetilde{\phi_{+s}^{\varepsilon}}= & (i \nabla+\varepsilon \vec{A})^{2} \widetilde{\phi^{\varepsilon}+s}+A_{0} \widetilde{\phi^{\varepsilon}+s} \\
& -\varepsilon \frac{1}{2} S^{k} B_{k} \widetilde{\phi_{+s}^{\varepsilon}}-i \frac{\varepsilon}{2} S^{k}\left(\partial_{k} A_{0}\right) \widetilde{\phi_{+l}^{\varepsilon}}
\end{aligned}
$$

The last terms on the r.h.s. which couple the system result from the $\gamma^{0} \gamma^{k}$-term in (2.21)

Using (1.17) and the orthogonality of $\Pi_{+}^{0}$ and $\Pi_{-}^{0} \quad$ we have $\phi_{+l}^{\varepsilon}=\Pi_{+}^{0} \phi^{\varepsilon}-i \frac{\varepsilon}{2} \gamma^{0} \gamma^{k} \partial_{k} \Pi_{+}^{0} \phi^{\varepsilon}+$ $O\left(\varepsilon^{2}\right)=O(1) \quad$ and $\quad \phi_{+s}^{\varepsilon}=+i \varepsilon / 2 \gamma^{0} \gamma^{k} \partial_{k} \Pi_{+}^{0} \phi^{\varepsilon}+$ $O\left(\varepsilon^{2}\right)=O(\varepsilon)$ in $L^{\infty}\left((0, T) ; L^{2}\left(\mathbb{R}^{3}\right)^{4}\right)$.

This yields finally $-i \varepsilon / 2 S^{k}\left(\partial_{k} A_{0}\right) \widetilde{\phi_{+s}^{\varepsilon}}=O\left(\varepsilon^{2}\right)$ in $L^{\infty}\left((0, T) ; L^{2}\left(\mathbb{R}^{3}\right)^{4}\right)$ and we can neglect this term in the $O(\varepsilon)$ approximation. A more heuristic approach to this issue is given e.g., in $[5,12]$. Note again that for the non-static case this "spinelectric field coupling" term actually involves both the electric and the magnetic field since $\vec{\nabla} A_{0}=-\vec{E}+\partial_{t} \vec{A}$. 
With proper initial data we can therefore define $\tilde{\varphi}_{+l}^{\varepsilon}$ as the 2-vector skipping the zero components of $\widetilde{\phi_{+l}^{\varepsilon}}$ (cp. (2.2)) and approximate the electronlarge component $\phi_{+}^{\varepsilon}$ by the solution of the Pauli equation (2.8) for the 2 -vector $\tilde{\varphi}_{+l}^{\varepsilon}$

Corollary 3.1 The 2-vector of the upper components of the $O\left(\varepsilon^{2}\right)$ approximation $\widetilde{\phi_{+}^{\varepsilon}}$ of the Dirac equation according to (2.21), (3.4) can be approximated up to terms of $O\left(\varepsilon^{2}\right)$ in $L^{\infty}\left((0, T) ; L^{2}\left(\mathbb{R}^{3}\right)^{4}\right)$ by the solution of the "usual Pauli equation" (2.8)

$$
i \partial_{t} \tilde{\varphi}_{+l}^{\varepsilon}=(-i \vec{\nabla}-\varepsilon \vec{A})^{2} \tilde{\varphi}_{+l}^{\varepsilon}-A_{0} \tilde{\varphi}_{+l}^{\varepsilon}-\varepsilon \frac{1}{2} \sigma^{k} B_{k} \tilde{\varphi}_{+l}^{\varepsilon}
$$

\section{HIGHER ORDER APPROXIMATIONS}

We can continue the Hilbert expansion (2.17) to higher orders in $\varepsilon$. This procedure can be justified by assuming more regularity on the electromagnetic potential and the initial data as a result of the following

Proposition 4.1 Let $A=\left(A_{0}, A_{1}, A_{2}, A_{3}\right) \in W^{1, \infty}$ $\left((0, T), W^{m, \infty}\left(\mathbb{R}^{3}\right)^{4}\right.$ and take initial data such that $\psi_{I}^{\varepsilon}=\psi^{\varepsilon}(t=0)=\phi^{\varepsilon}(t=0)=O(1) \in H^{m+1} \quad\left(\mathbb{R}^{3}\right)^{4}$ and $\pi_{-}^{\varepsilon} \psi_{I}^{\varepsilon}=\phi_{-}^{\varepsilon}(t=0)=O\left(\varepsilon^{2}\right) \in H^{m}\left(\mathbb{R}^{3}\right)^{4}$. Then

(i)

$$
\left\|\partial_{t} \phi_{-}^{\varepsilon}(t, x)\right\|_{H^{m-2}\left(\mathbb{R}^{3}\right)^{4}}=O(1), \quad \forall t \in(0, T)
$$

(ii)

$$
\text { with a bound analogous to (3.6), and }
$$

$$
\left\|\phi_{-}^{\varepsilon}(t)\right\|_{H^{m}\left(\mathbb{R}^{3}\right)^{4}}=O\left(\varepsilon^{2}\right), \quad \forall t \in(0, T)
$$

Proof The crucial estimates (3.6), (3.7) and (3.8) in the proof of Theorem 3.1 can easily be generalized to $\left.H^{m-2}(\mathbb{R})^{3}\right)^{4}$ and $\left.H^{m}(\mathbb{R})^{3}\right)^{4}$ estimates. The essential idea is the use of "finite induction", i.e., by assuming the estimate to hold for $m-1$ and show that it then holds for $m$ by e.g., multiplying the equation by $m$-th derivatives, integrating and so on. Since we have "conservation of regularity of degree $m$ " due to Lemma 2.1 the respective assumptions on the initial data of the corollary are sufficient to justify these manipulations.

In next order we have $O\left(\varepsilon^{2}\right)$ :

$$
\begin{aligned}
i \partial_{t} \phi_{+}^{2}= & -\frac{\Delta}{2} \phi_{+}^{2}-A_{0} \phi_{+}^{2} \\
& +\frac{i}{2} \partial_{k} A_{l} \gamma^{k} \gamma^{l} \phi_{+}^{1}-i A_{k} \partial_{k} \phi_{+}^{1} \\
& -\frac{i}{2} \gamma^{0} \gamma^{k} \partial_{k} A_{0} \phi_{+}^{1} \\
& -\frac{1}{8}\left(D_{x}\right)^{4} \phi_{+}^{0}-\frac{1}{2} A_{k} A_{l} \gamma^{k} \gamma^{l} \phi_{+}^{0} \\
& +\frac{\gamma^{k}}{4}\left\{\Delta\left(A_{k} \phi_{+}^{0}\right)+A_{k} \Delta \phi_{+}^{0}\right\} \\
& +\frac{\gamma^{0}}{4}\left\{\Delta\left(A_{0} \phi_{+}^{0}\right)-A_{0} \Delta \phi_{+}^{0}\right\} \\
\phi_{-}^{2}= & -\frac{1}{2} A_{k} \gamma^{0} \gamma^{k} \phi_{+}^{0}
\end{aligned}
$$

We define $\phi^{\varepsilon}:=\phi_{+}^{0}+\varepsilon \phi_{+}^{1}+\varepsilon^{2} \phi_{+}^{2}$ and obtain its equation by adding (2.18), (2.19) to the above equation for $\phi_{+}^{2}$. Taking into account that $\varepsilon \phi_{+}^{0}+\varepsilon^{2} \phi_{+}^{1}=$ $\varepsilon \phi^{\varepsilon}+O\left(\varepsilon^{3}\right)$ and $\varepsilon^{2} \phi_{+}^{0}=\varepsilon^{2} \phi+O\left(\varepsilon^{3}\right)$ we obtain the $O\left(\varepsilon^{3}\right)$ approximation:

$$
\begin{aligned}
i \partial_{t} \phi^{\varepsilon}= & -\frac{\Delta}{2} \phi^{\varepsilon}+-A_{0} \phi^{\varepsilon} \\
& +\varepsilon \frac{i}{2} \partial_{k} A_{l} \gamma^{k} \gamma^{l} \phi^{\varepsilon}-\varepsilon i A_{k} \partial_{k} \phi^{\varepsilon} \\
& +\varepsilon \frac{i}{2} \gamma^{0} \gamma^{k} \partial_{k} A_{0} \phi^{\varepsilon} \\
& -\frac{\varepsilon^{2}}{8}\left(D_{x}\right)^{4} \phi^{\varepsilon}-\frac{\varepsilon^{2}}{2} A_{k} A_{l} \gamma^{k} \gamma^{l} \phi^{\varepsilon} \\
& +\varepsilon^{2} \frac{\gamma^{k}}{4}\left\{\Delta\left(A_{k} \phi^{\varepsilon}\right)+A_{k} \Delta \phi^{\varepsilon}\right\} \\
& +\varepsilon^{2} \frac{\gamma^{0}}{4}\left\{\Delta\left(A_{0} \phi^{\varepsilon}\right)-A_{0} \Delta \phi^{\varepsilon}\right\}
\end{aligned}
$$

The $O\left(\varepsilon^{2}\right)$ terms correspond to the terms that occur in the Foldy-Wouthousen approach and can be interpreted as follows:

The term $\left(D_{x}\right)^{4} / 8 \phi^{\varepsilon}$ is the "mass term" $[4,10,11]$, i.e., the next correction of the relativistic kinetic 
energy which follows immediately from the explicit $O\left(\varepsilon^{2}\right)$ term in (2.12).

The diagonal of the next term is $\varepsilon^{2}|\vec{A}|^{2} \phi^{\varepsilon}$ precisely the term that has to be added for the "Pauli-operator" as in (2.8) or (2.21). The offdiagonal vanishes since the Dirac matrices anticommute, i.e., $\gamma^{\mu} \gamma^{\nu}+\gamma^{\nu} \gamma^{\mu}=0$.

The last term can be rewritten as $\gamma^{0} / 4\left\{\Delta A_{0}\right.$ $\left.\phi^{\varepsilon}-\nabla A_{0} \cdot \nabla \phi^{\varepsilon}\right\}$ and corresponds to the "Darwin term" related to the "zitterbewegung" [10].

By separating again the upper component $\phi_{l}^{\varepsilon}:=\Pi_{+}^{0} \phi^{\varepsilon}$ and the lower component $\phi_{s}^{\varepsilon}:=\Pi_{-}^{0} \phi^{\varepsilon}$ in complete analogy to (3.9) we can again see that the terms containing the "anti blockdiagonal" matrices $\gamma^{k}$ are implicitly one order smaller.

Hence the term $\varepsilon^{2} \gamma^{k} / 4\left\{\Delta\left(A_{k} \phi^{\varepsilon}\right)+A_{k} \Delta \phi^{\varepsilon}\right\}$ occurs at order $O\left(\varepsilon^{3}\right)$ in the equation for $\phi_{l}^{\varepsilon}$ and can be neglected in the second order approximation.

The term $\varepsilon i / 2 \gamma^{0} \gamma^{k} \partial_{k} A_{0} \phi^{\varepsilon}$ occurring also in (3.10) which we already have shown to be actually of order $O\left(\varepsilon^{2}\right)$ has to be taken into account now. This term can be reformulated like in [10] but for the time dependent case we intrinsically have the difference to the static case that this "spin-electric field coupling" or "spin-orbit coupling" always involves also the magnetic field since $\vec{\nabla} A_{0}=-\vec{E}(t, x)+\partial_{t} \vec{A}$. In the case of extremely rapidly varying magnetic fields this $O\left(\varepsilon^{2}\right)$ term can lead to a significant difference with the relativistic corrections of the Foldy-Wouthousen approach where time derivatives of the electromagnetic potential do not occur.

The procedure of the Hilbert expansion can be continued to third and higher orders. With each order, of course, more derivatives of the spinor and the potential occur. In order to justify the formal development we have to ask for more and more regularity of the initial data and the potential as stated in Proposition 4.1 Thus we can avoid distributional spaces and stay in the framework of $L^{2}\left(\mathbb{R}^{3}\right)^{4}$.

\section{Acknowledgement}

Financial support by the DAAD-PROCOPE, by the European TMR "Marie Curie-Fellowship" contract \# ERBFMBICT961125 and by the Austrain FWF "Erwin-Schrödinger Fellowship" project \# J01131-MAT are acknowledged.

\section{References}

[1] Arnold A. and Steinrück, H. (1989). "The 'electromagnetic" Wigner equation for an electron with spin", ZAMP, 40(6), 793-815.

[2] Armstrong, L. and Feneuille, S. (1974). Adv. Atom. Mol. Phys., 10(1).

[3] Bechouche, P., Mauser, N. J. and Poupaud, F. (1998). "(Semi)-nonrelativistic limits of the Dirac equation with external time-dependent electromagnetic field", Comm. Math. Phys., 197, 405-425.

[4] Chang, Ch., Pelissier, M. and Durand, Ph. (1986) "regular Two-component Pauli-like Effective Hamiltonians in Dirac Theory", Phys. Scripta., 34, 394-404.

[5] Dirac, P. A. M. (1958). "Principles of Quantum Mechanics", Oxford University Press, 4th ed., London.

[6] Esteban, M. J. and Séré, E. (1996). "Existence and multiplicity of solutions for linear and nonlinear Dirac problems", preprint No. 9542, CEREMADE, Univ. Paris IX.

[7] Foldy, L. L. and Wouthuysen, S. A. (1950). "On the Dirac theory of Spin 1/2 Particles and its Nonrelativistic Limit", Phys. Rev., 78, 29-36.

[8] Gérard, P., Markowich, P. A., Mauser, N. J. and Poupaud, F. (1997). "Homogenization Limits and Wigner Transforms", Comm. Pure and Appl. Math., 50, 321-377.

[9] Hunziker, W. (1975). "On the nonrelativistic limit of the Dirac Theory", Comm. Math. Phys., 40, 215-222

[10] Itzykson, C. and Zuber, J. B. (1985). "Quantum Field Theorie", McGraw-Hill, New York.

[11] Landau, L. and Lifschitz (1971). Vol. III, "Quantenmechanik", Vol. IVa, "Relativistische Quantenmechanik", Akademie-Verlag, 2nd ed., Berlin.

[12] Schiff (1968). "Quantum Mechanics", McGraw-Hill, 3rd ed., New York.

[13] Thaller, B. (1992). "The Dirac Equations", Springer, New York-Wien.

[14] White, G. B. (1990). "Splitting of the Dirac operator in the nonrelativisitic limit", Ann. Inst. Henri Poincare, Phys. Theor., 40, 109-121.

\section{Authors' Biography}

Norbert Julius Mauser, born 3.8.1964, studied Technical Mathematics and Technical Physics at the TU Vienna and Astronomie at the Univ. Vienna. He obtained his Ph.D. in Mathematics in 1994 at the TU-Vienna (advisor P.A. Markowich).

From $91-96$ he worked at the TU-Berlin. 199394 he was research consultant at the CRS4 in Cagliari, Italy, and he held postdoc positions at 
the Univ. Nice, France (96-97 as a Marie Curie Fellow) and at Courant Institute, NY (98-99 as an Erwin Schrödinger Fellow).

His main fields of interest are P.D.E. and Applied Mathematics where he has worked on modelling, analysis and numerics of quantum semiconductor devices. His work on asymptotic limits includes fundamental contributions on the use of Wigner transforms for semiclassical and homogenization limits. 

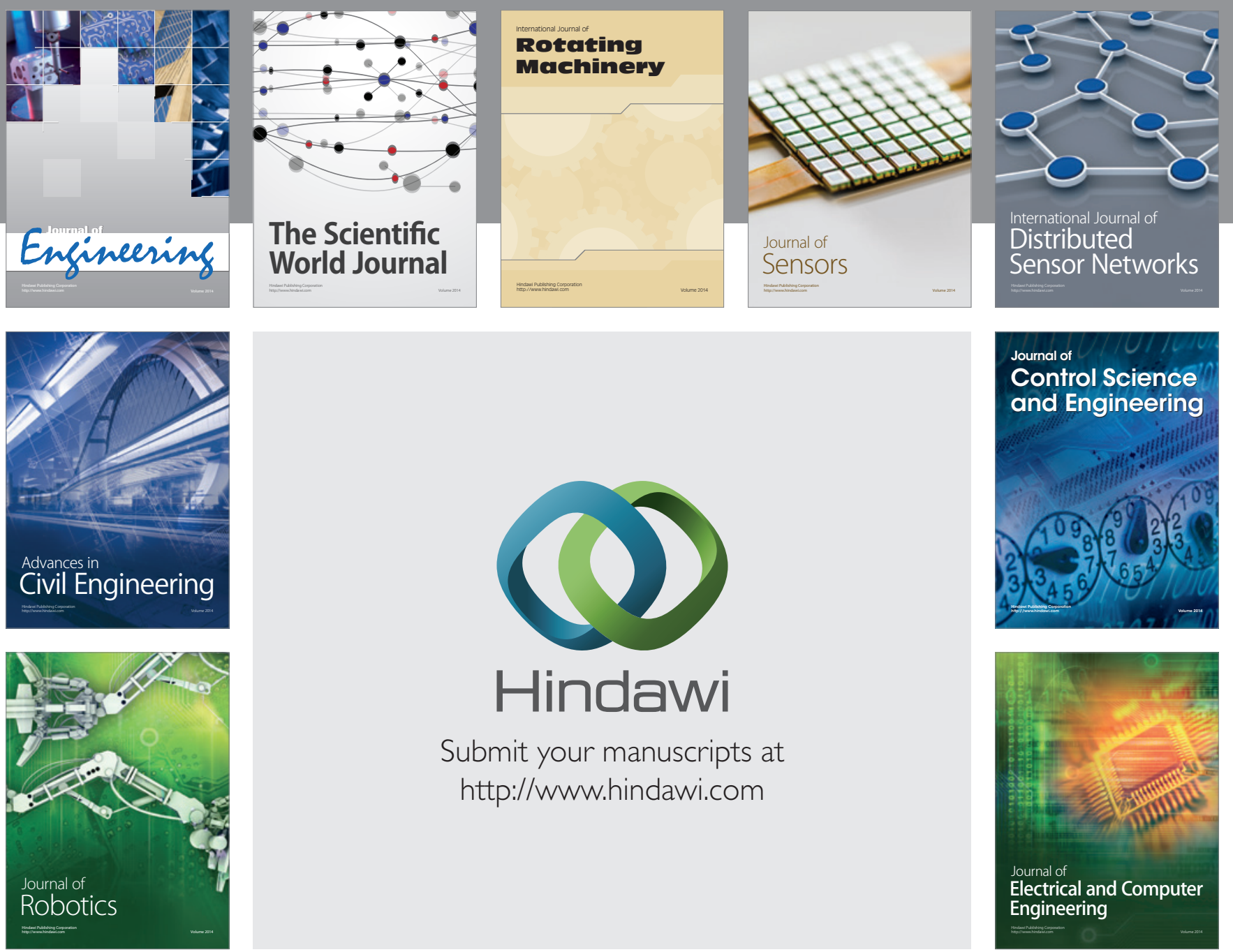

Submit your manuscripts at

http://www.hindawi.com
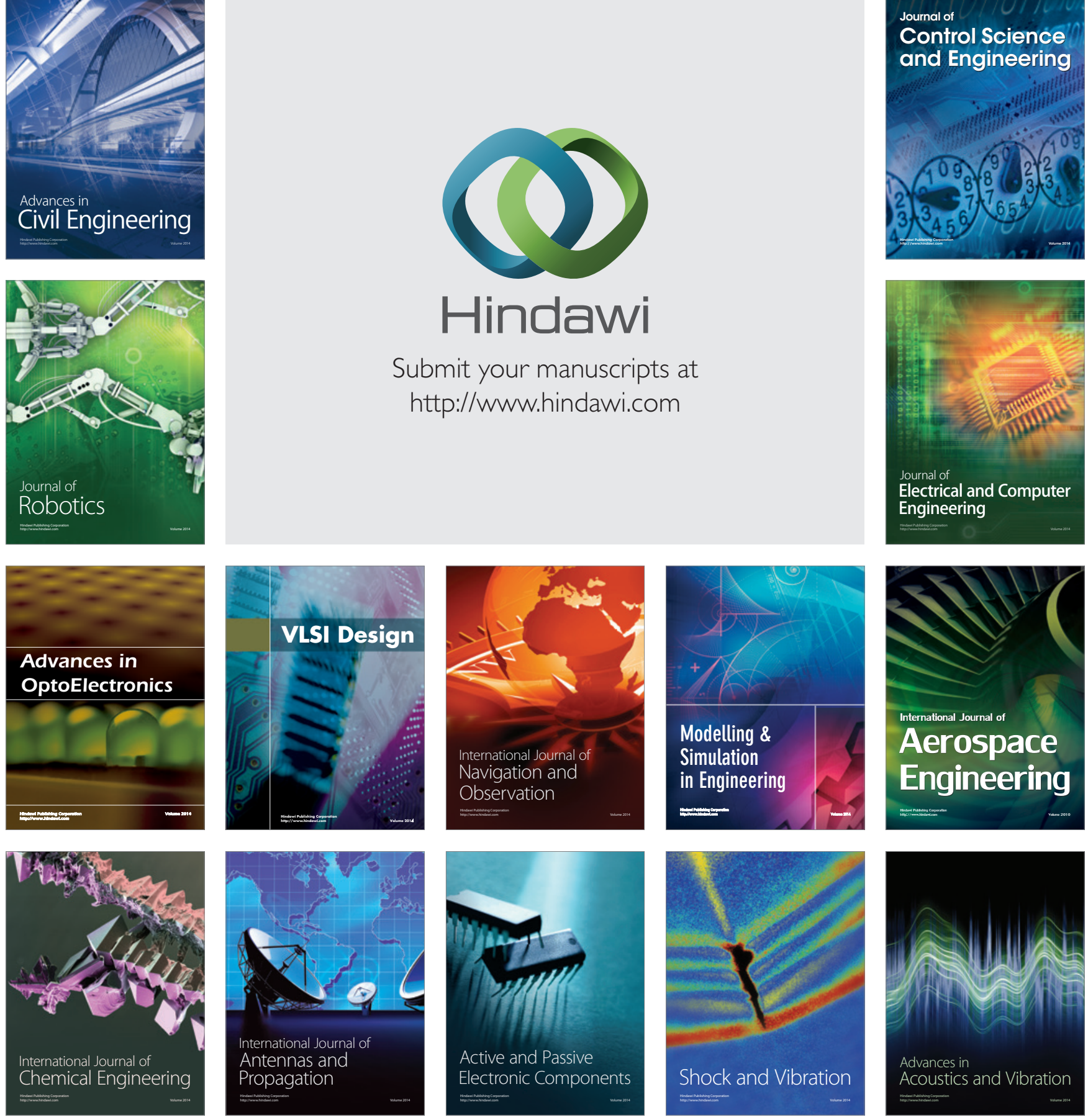